\title{
Magnetism in $\mathrm{UCo}_{0.88} \mathrm{Ru}_{0.12} \mathrm{Ge}$ Studied by Polarized Neutrons
}

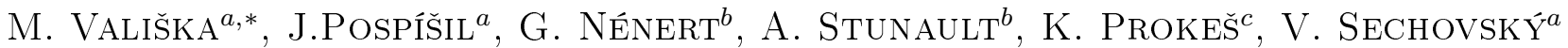 \\ ${ }^{a}$ Faculty of Mathematics and Physics, Charles University, DCMP, Ke Karlovu 5, CZ-12116 Praha 2, Czech Republic \\ ${ }^{b}$ Institut Laue Langevin, 6 rue Jules Horowitz, 38042 Grenoble, France \\ ${ }^{c}$ HZB Berlin, Hahn-Meitner Platz 114109 Berlin, Germany
}

\begin{abstract}
UCoGe is an archetype of coexisting weak itinerant $5 f$-electron ferromagnetism $\left(T_{C} \sim 3 \mathrm{~K}, \mu_{s}=0.03 \mu_{\mathrm{B}} /\right.$ f.u. $)$ and superconductivity $\left(T_{S C} \sim 0.6 \mathrm{~K}\right)$ at ambient pressure. The Ru substitution for Co leads to an initial sharp increase of the $T_{C}$ up to the maximum of $T_{C}=8.5 \mathrm{~K}$ for $x=0.12$, increase of spontaneous magnetic moment and suppression of superconductivity. We have grown the $\mathrm{UCo}_{0.88} \mathrm{Ru}_{0.12} \mathrm{Ge}$ single crystal and studied magnetization along the principal crystallographic axes. To see the microscopic background of these findings we performed a polarized neutron diffraction experiment on D3 diffractometer in ILL. We have found that the Co and U moments are parallel in $\mathrm{UCo}_{0.88} \mathrm{Ru}_{0.12} \mathrm{Ge}$, in contrast to the antiparallel configuration in UCoGe. This is probably the reason of the spontaneous magnetization increase with $\mathrm{Ru}$ doping.
\end{abstract}

DOI: 10.12693/APhysPolA.126.330

PACS: 74.70.Tx, 61.05.fm, 75.25.-j

\section{Introduction}

Former studies described UCoGe as a compound with paramagnetic ground state $[1,2]$. Breakthrough came with the report of Huy et al. [3], presenting this compound as a new member of the unique family of ferromagnetic superconductors. UCoGe has Curie temperature of $3 \mathrm{~K}$ and very low spontaneous magnetic moment of $0.03 \mu_{\mathrm{B}}$ [3]. Transition to the superconducting state is found at $T_{S C}=0.7 \mathrm{~K}[3]$ at ambient pressure. Results on magnetic properties of UCoGe published recently show large discrepancies between experimental data and results of some of the theoretical calculations. All three theoretical works predict a small total magnetic moment on the U site $\left(\sim 0.1 \mu_{\mathrm{B}}\right)$ which is caused by almost complete cancellation of the antiparallel spin and orbital moment [4-6]. All calculations provide also a net magnetic moment on the Co site, but differ in the mutual orientation of the $\mathrm{U}$ and Co moments, respectively. While Samsel-Czekala et al. [5] and de la Mora et al. [6] come with the parallel alignment, enhancing the total magnetic moment, Diviš [4] predicts antiparallel alignment. The proposed scenario, including the antiparallel configuration, has been subsequently confirmed by polarized neutron diffraction study. Prokeš et al. [7] revealed negative magnetic density on the Co site and the positive value on $\mathrm{U}$. It confirms that magnetic state of UCoGe is formed by Co moments, as well. Magnetic state of this compound was also studied in the frame of doping of other transition metal on the Co site. Pospišil et al. [8] showed, that both the $T_{C}$ and magnetic moment of the $\mathrm{U}(\mathrm{Co}, T)$ Ge system is enhanced by substituting $\mathrm{Fe}$ or $\mathrm{Ru}$ for Co. Closer study of the $\mathrm{UCo}_{1-x} \mathrm{Fe}_{x}$ Ge alloy in broad concentration range showed that initial rapid increase of

*corresponding author; e-mail: michal.valiska@gmail.com the $T_{C}$ and magnetic moment is consequently followed by steady suppression of ferromagnetic order and the whole system ends in the Quantum Critical Point [9].

In this manuscript we present results of our study of the influence of Ru substitution for Co on the evolution of magnetic properties of the $\mathrm{UCo}_{1-x} \mathrm{Ru}_{x}$ Ge performed on polycrystalline samples, and the details of magnetism in the $\mathrm{UCo}_{0.88} \mathrm{Ru}_{0.12} \mathrm{Ge}$ single crystal, the composition with the maximum $T_{C}$.

\section{Results}

We have prepared series of polycrystalline samples with various $\mathrm{Ru}$ concentration in the range of $x=0.01$ 0.9 . All these samples were prepared by arc-melting of stoichiometric amounts of elements (purity of Co $4 \mathrm{~N} 5$, Ge $5 \mathrm{~N}$ and $\mathrm{Ru} 3 \mathrm{~N} 5$ ) under protective Ar atmosphere. Each sample has been separately wrapped into the tantalum foil (purity $4 \mathrm{~N}$ ) sealed in a quartz tube under the vacuum of $1 \times 10^{-6}$ mbar, subsequently annealed at $885^{\circ} \mathrm{C}$ for 14 days and then slowly cooled down to room temperature to avoid internal stresses. The structure and composition of all the samples have been checked by Xray powder diffraction and EDX microprobe analysis, respectively.

The magnetization curves measured on the polycrystalline samples (Fig. 1) revealed the expected increase of spontaneous magnetic moment up to the $\sim 0.11 \mu_{\mathrm{B}}$ for $x \approx 0.1$. Higher concentrations of Ru lead to the subsequent lowering of spontaneous magnetic moment.

We focused our interest on this concentration region with the enhanced magnetic moment and $T_{C}$. We prepared a single crystal with the composition of $\mathrm{UCo}_{0.88} \mathrm{Ru}_{0.12}$ Ge by floating zone method. The measurements of magnetization along the three crystallographic axes showed large magnetocrystalline anisotropy, with the $c$-axis as a magnetic easy axis. The spontaneous magnetic moment along this axis was $\sim 0.21 \mu_{\mathrm{B}}(\sim 85 \%$ higher than that for parent UCoGe). 


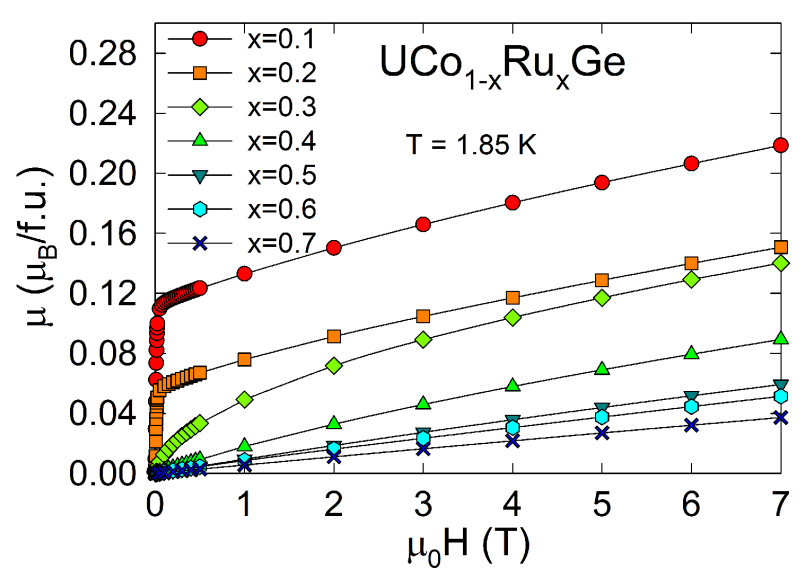

Fig. 1. Magnetization curves measured for various concentrations of Ru.

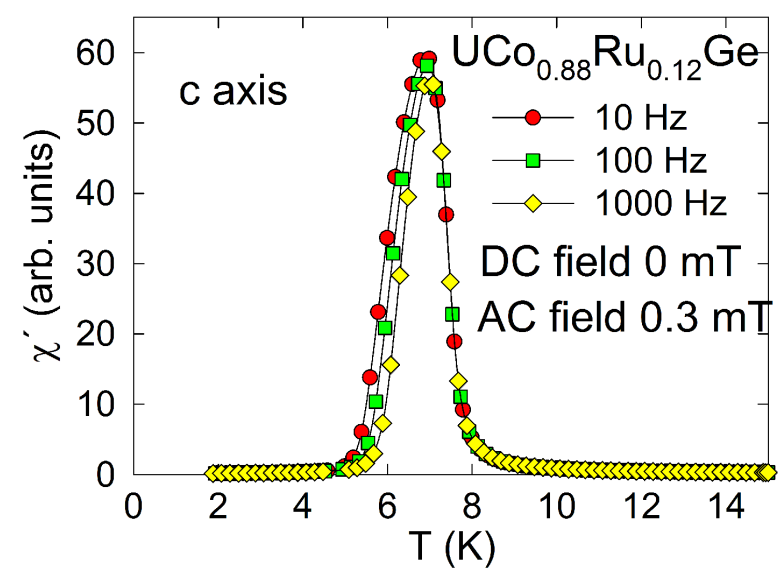

Fig. 2. Real part of AC susceptibility measured on $\mathrm{UCo}_{0.88} \mathrm{Ru}_{0.12} \mathrm{Ge}$ single crystal.

The AC susceptibility measurement showed a weak frequency dependence of the anomaly at $T_{C} \approx 7.6 \mathrm{~K}$ (see Fig. 2). It can be connected with the domain wall formation as in the similar case of the $\mathrm{U}_{3} \mathrm{Co}_{4} \mathrm{Ge}_{7}$ [10].

Polarized neutron diffraction study performed on this crystal at $1.65 \mathrm{~K}$ (well below $T_{C}$ ) revealed the parallel alignment of magnetic moments on the $\mathrm{Co}(\mathrm{Ru})$ and $\mathrm{U}$ site. In the field of $9 \mathrm{~T}$ applied along the $c$ axis the magnetic moment on the $\mathrm{Co}(\mathrm{Ru})$ site $\mu_{C o}=0.069 \mu_{\mathrm{B}}$ and on the $\mathrm{U}$ site $\mu_{U}=0.257 \mu_{\mathrm{B}}$. This result is in contrast to the antiparallel configurations in $\mathrm{UCoGe}$, in which the $\mu_{C o}=-0.27 \mu_{\mathrm{B}}$ and $\mu_{U}=0.32 \mu_{\mathrm{B}}$. These results suggest that the reorientation of the magnetic moment on the $\mathrm{Co}(\mathrm{Ru})$ site is the main reason of the initial increase of the total magnetic moment with increasing Ru concentration in $\mathrm{UCo}_{1-x} \mathrm{Ru}_{x} \mathrm{Ge}$, while the magnetic moment on $\mathrm{U}$ site may be little influenced.

\section{Conclusions}

We carried out magnetization measurements on the polycrystalline samples of the $\mathrm{UCo}_{1-x} \mathrm{Ru}_{x} \mathrm{Ge}$ series. These results showed initial increase of the total magnetic moment and $T_{C}$ followed by steady decrease of these parameters for higher $\mathrm{Ru}$ concentration $(x>0.1)$. In order to find a mechanism responsible for the initial rapid increase we performed polarized neutron diffraction on the high quality $\mathrm{UCo}_{0.88} \mathrm{Ru}_{0.12} \mathrm{Ge}$ single crystal. We have observed a change in the mutual orientation of magnetic moments on the $\mathrm{U}$ and Co site which seems to be the primary reason of the evolution of magnetism in low $\mathrm{Ru}$ concentrations. The antiparallel orientation for $\mathrm{UCoGe}$ gives a lower moment of $\sim 0.03 \mu_{\mathrm{B}}[3]$, while the parallel alignment for the doped $\mathrm{UCo}_{0.88} \mathrm{Ru}_{0.12} \mathrm{Ge}$ leads to the spontaneous magnetic moment of $\sim 0.21 \mu_{\mathrm{B}}$.

\section{Acknowledgments}

This work was supported by the Charles University Grant Agency Project \# 719612 and Czech Science Foundation no. P204/12/P418. Experiments performed in the Magnetism and Low Temperature Laboratories at Charles University in Prague were supported by Project No. LM2011025. Neutron diffraction experiments in ILL, Grenoble, were performed within Project No. LG11024.

\section{References}

[1] R. Troc, V.H. Tran, J. Magn. Magn. Mater. 73, 389 (1988).

[2] K.H.J. Buschow, E. Bruck, R.G. van Wierst, F.R. de Boer, L. Havela, V. Sechovsky, P. Nozar, E. Sugiura, M. Ono, M. Date, A. Yamagishi, J. Appl. Phys. 67, 5215 (1990).

[3] N.T. Huy, A. Gasparini, D.E. de Nijs, Y. Huang, J.C.P. Klaasse, T. Gortenmulder, A. de Visser, A. Hamann, T. Gorlach, H. von Lohneysen, Phys. Rev. Lett. 99, 067006 (2007).

[4] M. Divis, Physica B: Condensed Matter 403, 2505 (2008).

[5] M. Samsel-Czekala, S. Elgazzar, P.M. Oppeneer, E. Talik, W. Walerczyk, R. Troc, J. Phys.: Condens. Matter 22, 015503 (2010).

[6] P. de la Mora, O. Navarro, J. Phys.: Condens. Matter 20, 285221 (2008).

[7] K. Prokes, A. de Visser, Y.K. Huang, B. Fak, E. Ressouche, Phys. Rev. B 81, 180407R (2010).

[8] J. Pospisil, J.P. Vejpravova, M. Divis, V. Sechovsky, J. Appl. Phys. 105, 07E114 (2009).

[9] K. Huang, J.J. Hamlin, R.E. Baumbach, M. Janoschek, N. Kanchanavatee, D.A. Zocco, F. Ronning, M.B. Maple, Phys. Rev. B 87, 054513 (2013).

[10] K. Uhlirova, M. Divis, J. Pospisil, S. Danis, V. Sechovsky, J. Phys. Soc. Jpn. 81, 094703 (2012). 